\title{
Different soliton solutions to the modified equal-width wave equation with Beta-time fractional derivative via two different methods
}

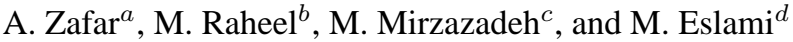 \\ ${ }^{a}$ Department of Mathematics, CUI, Vehari Campus, Pakistan. \\ ${ }^{b}$ Department of Mathematics \& Statistics, ISP Multan, Pakistan. \\ ${ }^{c}$ Department of Engineering Sciences \&, East of Guilan, University of Guilan, \\ P.C. 44891-63157, Rudsar-Vajargah, Iran. \\ ${ }^{d}$ Department of Mathematics \& University of Mazandaran, Babolsar, Iran. \\ *e-mail: mostafa.eslami@umz.ac.ir
}

Received 5 December 2020; accepted 13 April 2021

\begin{abstract}
In this paper, different types of solitary wave solutions for the modified equal-width wave (MEW) equation with beta time derivative is obtained by implementing the extended Jacobi's elliptic function expansion method and the Kudryashov method. The secured solutions are in the form of dark, bright, singular solitons and other soliton type solutions. The obtained solutions are verified through symbolic soft computation. The solutions also suggest that these two methods are effective, straight forward and reliable as compared to other methods. The obtained results can be used in describing the substantial understanding of the studious structures as well as other related non-linear physical structures.
\end{abstract}

Keywords: Modified equal width equation; beta derivative; soliton solutions.

DOI: https://doi.org/10.31349/RevMexFis.68.010701

\section{Introduction}

Solitary wave theory has gained much importance because of its use in the field of applied physics. Waves are generated when some disturbance occur in the phenomenon. Soliton interactions occur where two or more than two solitons come close enough to each other. Because solitons present themselves as tiny, confined energy bundles, it is said that they show the particle-like characteristics of a given system. One of the most important technical applications of solitons is their use in optical fibers to carry digital information. In electromagnetism, solitons are studied as the transverse wave that travels between two strips of superconducting metals. Beyond these, solitons have been found to be useful in many applications across different areas of science and engineering. Solitons are governed by nonlinear Schrödinger equations, which represent the physical phenomena as models using non-linear partial differential equations (NLPDEs). There are many analytical schemes that have been constructed to solve such non-linear partial differential equations. For instance, Biswas and Alqahtani have determined the two types of bright solitons of perturbed Gerdjikov-Ivanov equation (PGIE) by using Semi-inverse Variational method [1]. Various solitons for some coupled evolution equations were explained in [2]. Exponential rational function scheme [3] was applied to find out the hyperbolic rational function type solitons of the Boussinesq fractional type models for some certain physical phenomenon. Periodic type solitons have been investigated, by implementing the variational principle method [4], for the KMN equation. Various optical soliton solutions in the fiber communication system have been obtained by employing the Riccati equation method [5] and spatio-temporal like optical solitons have been determined in Ref. [6]. The famous Biswas and Arshed model with nonlinearity factor " $n$ " has been explored by employing the modified extended tanh expansion technique [7]. In this paper we are interested in investigation of an important model named modified equal-width (MEW) equation in the beta derivative sense. The aforementioned equation also finds an important role in plasma physics and fluid dynamics.

This equation has been solved by different analytical methods such as: the tanh-function method [8,9], the ansatz and improved $\left(G^{\prime} / G\right)$-expansion methods [10]. But the extended Jacobi elliptic expansion function method and Kudryashov method have not been exercised for the above mentioned model with a fractional beta derivative operator. These methods have also been used to explore different models in different articles, see for example [11-15]. Furthermore, by applying the Kudryashov scheme, exact solutions to the fractional and classical GEW-Burgers equations have been determined in [16]. Hosseini et al. obtained the soliton solutions of the Perturbed Gerdjikov-Ivanov equation by employing the Kudryashov technique [17-19]. Moreover, in different applied fields, physical model equations using the novel beta derivative and Atangana's-conformable derivative operators have been investigated via distinct techniques [20-24].

The primary prospect of this paper is to determine the wave form solutions of the MEW wave equation with betatime derivative based on the two different methods, the extended Jacobi's elliptic function expansion method and the Kudryashov method. 


\section{2. $\beta$-Derivative and it's properties}

Definition: Suppose $g(\theta)$ is a function that is defined $\forall$ non-negative $\theta$. Therefore, the beta-time fractional derivative of the function $\mathrm{g}$ of power $\beta$ is given as [25]

$$
D^{\beta}(g(\theta))=\frac{d^{\beta} g(\theta)}{d \theta^{\beta}}=\lim _{\epsilon \rightarrow 0} \frac{g\left(\theta+\epsilon\left(\theta+\frac{1}{\Gamma(\beta)}\right)^{1-\beta}\right)-g(\theta)}{\epsilon}, \quad 0<\beta \leq 1 .
$$

Few useful features of the Beta-time fractional derivative are given follows [26-30]

Theorem:

Suppose $f(\theta)$ and $g(\theta)$ are the $\beta$-time differentiable functions $\forall \theta>0$ and $\beta \in(0,1]$. Then

$$
\begin{array}{ll}
\text { i. } & D^{\beta}(a f(\theta)+b g(\theta))=a D^{\beta}(f(\theta))+b D^{\beta}(g(\theta)), \forall a, b \in R . \\
\text { ii. } & D^{\beta}(f(\theta) g(\theta))=g(\theta) D^{\beta}(f(\theta))+f(\theta) D^{\beta}(g(\theta)) . \\
\text { iii. } & D^{\beta}\left(\frac{f(\theta)}{g(\theta)}\right)=\frac{g(\theta) D^{\beta}(f(\tau))-f(\theta) D^{\beta}(g(\theta))}{(g(\theta))^{2}} . \\
\text { iv. } & D^{\beta}(f(\theta))=\left(\theta+\frac{1}{\Gamma(\beta)}\right)^{1-\beta} \frac{d f(\theta)}{d \theta} .
\end{array}
$$

\section{Description of Strategies}

\subsection{Explanation of the extended Jacobi's elliptic function expansion method}

Here, we explain the general steps of the extended Jacobi's elliptic function expansion scheme [11]: Assume the below travelling wave equation in the form of PDE:

$$
G\left(q_{t}, q^{2} q_{t}, q_{x}, q_{t t}, q_{x x}, q_{x t}, \ldots\right)=0
$$

here $q=q(x, t)$. Let us assume the propagational waves transformations:

$$
q(x, t)=Q(\eta), \quad \eta=x-\mu t
$$

where $\mu$ characterizes the soliton speed. Inserting Eq. (2) into Eq. (1), leads to the non-linear ordinary differential equation (NODE):

$$
F\left(Q(\eta), Q^{2}(\eta) Q^{\prime}(\eta), Q^{\prime \prime}(\eta), Q^{\prime \prime \prime}(\eta), \ldots\right)=0 .
$$

The above obtained Eq. (3) has the following type of solutions by applying the extended Jacobi’s elliptic function expansion scheme:

$$
Q(\eta)=\sum_{j=-M}^{N} \alpha_{j} Y^{j}(\eta)
$$

where $M, N, \alpha_{j}(j=-M, \ldots, N)$ are unknowns to be found later while $Y$ represents the Jacobi's elliptic function, namely, $Y=Y(\eta)=s n \eta=s n(\eta, m)$ or $c n(\eta, m)$ or $d n(\eta, m)$ where $0<m<1$ is the amplitude of Jacobi's elliptic functions. The values of $M$ and $N$ may be found by using the balance technique of highest derivative and nonlinear term in Eq. (3). After that, substituting Eq. (4) into the Eq. (3), we obtain a system of algebraic equation in terms of $\alpha_{j}(j=-M, \ldots, N)$. Now by using Mathematica, we can solve the gained system of algebraic equations for $\alpha_{j}$. By plugging these obtained values into Eq. (4), the general form of Jacobi's elliptic function solution of Eq. (1) can be given. When $m \rightarrow 1$, the Jacobi functions are transformed into hyperbolic functions given as:

$$
s n(\eta, m) \rightarrow \tanh (\eta), \quad c n(\eta, m) \rightarrow \operatorname{sech}(\eta) \quad \text { and } \quad d n(\eta, m) \rightarrow \operatorname{sech}(\eta) .
$$

\subsection{Explanation of the Kudryashov method}

The procedure of Kudryashov method is explained in the steps below [17]:

Step 1:

Suppose Eqs. (1), (2) and (3).

Step 2: 
Consider the solutions of Eq. (3) are of the type:

$$
Q(\eta)=\sum_{j=0}^{m} \alpha_{j} \phi^{j}(\eta)
$$

Here, $\alpha_{j}(j=0,1,2,3, \ldots, m)$ are the unknowns with $\alpha_{j} \neq 0$ to be found. The positive integer $m$ will be calculated using the homogenous balance technique.

The function $\phi(\eta)$ satisfies the auxiliary differential equation

$$
\left(\phi^{\prime}(\eta)\right)^{2}=\phi^{2}(\eta)\left(1-d \phi^{2}(\eta)\right)
$$

Eq. (6) gives the following solution.

$$
\phi(\eta)=\frac{4 a}{\left(4 a^{2}-d\right) \sinh (\eta)+\left(4 a^{2}+d\right) \cosh (\eta)}, d=4 a b, a \text { and } b \text { are constants. }
$$

Step 3: By combining Eqs. (3), (5), and (6), summing up all coefficients of the same order on $\phi(\eta)$, and taking each coefficient equal to zero, we can solve for the algebraic expressions involving $\alpha_{j}, \mu$, and other parameters.

Step 4: Putting the above finding results of the unknowns with the solutions of the Eq. (7), we get the solutions of the non-linear partial differential equation Eq. (1).

\section{Model description and it's mathematical analysis}

Consider the modified equal width wave (MEW) equation [10] with beta-time fractional derivative given as

$$
\frac{\partial^{\beta} q}{\partial t^{\beta}}+\theta \frac{\partial q^{3}}{\partial x}-\rho \frac{\partial^{2}}{\partial x^{2}}\left(\frac{\partial^{\beta} q}{\partial t^{\beta}}\right)=0
$$

Here $q=q(x, t)$ is the wave profile while $\theta$ and $\rho$ are the parameters. Let us assume the following travelling wave transformation:

$$
q(x, t)=Q(\eta), \quad \eta=\omega x-\frac{\lambda}{\beta}\left(t+\frac{1}{\Gamma(\beta)}\right)^{\beta} .
$$

Here $\omega$ and $\lambda$ are the constants. By using Eq. (9) into the Eq. (), we get the following ODE

$$
-\lambda Q^{\prime}+\theta \omega\left(Q^{3}\right)^{\prime}+\rho \lambda \omega^{2} Q^{\prime \prime \prime}=0 .
$$

After integrating Eq. (10) once with respect to $\eta$, we get

$$
-\lambda Q+\theta \omega Q^{3}+\rho \lambda \omega^{2} Q^{\prime \prime}=0
$$

\subsection{Solutions with the extended Jacobi's elliptic function expansion method}

Balancing the terms $Q^{\prime \prime}$ and $Q^{3}$ in Eq. (11), we get $M=N=1$. So Eq. (4) reduces to

$$
Q(\eta)=\alpha_{-1} Y^{-1}(\eta)+\alpha_{0}+\alpha_{1} Y(\eta)
$$

Case 1: If $Y=Y(\eta)=\operatorname{sn}(\eta, m)$ Eq. (12) becomes:

$$
Q(\eta)=\alpha_{-1} s n^{-1}(\eta, m)+\alpha_{0}+\alpha_{1} s n(\eta, m) .
$$

By substituting Eq. (13) into Eq. (11), we obtain the solution sets given below.

Set 1:

$$
\left\{\alpha_{-1}=\mp \frac{\sqrt{2} \sqrt{\lambda} \sqrt{\rho}}{\sqrt{\theta} \sqrt[4]{-(m+1) \rho}}, \quad \alpha_{0}=0, \quad \alpha_{1}=0, \quad \omega=\frac{-1}{\sqrt{-(m+1) \rho}}\right\} .
$$

By using Eqs. (13) and (14) into Eq. (9), we get

$$
q(x, t)=\mp \frac{\sqrt{2} \sqrt{\lambda} \sqrt{\rho}}{\sqrt{\theta} \sqrt[4]{-(m+1) \rho}} s n^{-1}\left(\frac{-x}{\sqrt{-(m+1) \rho}}-\frac{\lambda}{\beta}\left[t+\frac{1}{\Gamma(\beta)}\right]^{\beta}\right) .
$$


Set 2:

$$
\left\{\alpha_{-1}=0, \quad \alpha_{0}=0, \quad \alpha_{1}=\mp \frac{\sqrt{2} \sqrt{\lambda} \sqrt{m} \sqrt{\rho}}{\sqrt{\theta} \sqrt[4]{-(m+1) \rho}}, \quad \omega=\frac{-1}{\sqrt{-(m+1) \rho}}\right\} .
$$

By using Eqs. (13) and (16) into Eq. (9), we get

$$
q(x, t)=\mp \frac{\sqrt{2} \sqrt{\lambda} \sqrt{m} \sqrt{\rho}}{\sqrt{\theta} \sqrt[4]{-(m+1) \rho}} \operatorname{sn}\left(\frac{-x}{\sqrt{-(m+1) \rho}}-\frac{\lambda}{\beta}\left[t+\frac{1}{\Gamma(\beta)}\right]^{\beta}\right) .
$$

Set 3:

$$
\left\{\alpha_{-1}=\mp \frac{i \sqrt{2} \sqrt{\lambda} \sqrt{\rho}}{\sqrt{\theta} \sqrt[4]{-(m+1) \rho}}, \quad \alpha_{0}=0, \quad \alpha_{1}=0, \quad \omega=\frac{1}{\sqrt{-(m+1) \rho}}\right\} .
$$

By using Eqs. (13) and (18) into Eq. (9), we get

$$
q(x, t)=\mp \frac{i \sqrt{2} \sqrt{\lambda} \sqrt{\rho}}{\sqrt{\theta} \sqrt[4]{-(m+1) \rho}} \operatorname{sn}^{-1}\left(\frac{x}{\sqrt{-(m+1) \rho}}-\frac{\lambda}{\beta}\left[t+\frac{1}{\Gamma(\beta)}\right]^{\beta}\right) .
$$

Set 4:

$$
\left\{\alpha_{-1}=0, \quad \alpha_{0}=0, \quad \alpha_{1}=\mp \frac{i \sqrt{2} \sqrt{\lambda} \sqrt{m} \sqrt{\rho}}{\sqrt{\theta} \sqrt[4]{-(m+1) \rho}}, \quad \omega=\frac{1}{\sqrt{-(m+1) \rho}}\right\} .
$$

By using Eqs. (13) and (20) into Eq. (9), we get

$$
q(x, t)=\mp \frac{i \sqrt{2} \sqrt{\lambda} \sqrt{m} \sqrt{\rho}}{\sqrt{\theta} \sqrt[4]{-(m+1) \rho}} \operatorname{sn}\left(\frac{x}{\sqrt{-(m+1) \rho}}-\frac{\lambda}{\beta}\left[t+\frac{1}{\Gamma(\beta)}\right]^{\beta}\right) .
$$

Set 5:

$$
\left\{\alpha_{-1}=\mp \sqrt{\frac{2 \rho \lambda}{\theta}} \sqrt[4]{\frac{-1}{(m-6 \sqrt{m}+1) \rho}}, \alpha_{0}=0, \alpha_{1}= \pm \sqrt{\frac{2 m \rho \lambda}{\theta}} \sqrt[4]{\frac{-1}{(m-6 \sqrt{m}+1) \rho}}, \omega=\frac{-\iota}{\sqrt{(m-6 \sqrt{m}+1) \rho}}\right\} .
$$

By using Eqs. (13) and (22) into Eq. (9), we get

$$
\begin{aligned}
q(x, t) & =\sqrt{2} \sqrt{\rho} \sqrt{\frac{\lambda}{\theta}} \sqrt[4]{-\frac{1}{(m-6 \sqrt{m}+1) \rho}}\left(\mp \operatorname{sn}^{-1}\left[\frac{-\iota x}{\sqrt{(m-6 \sqrt{m}+1) \rho}}-\frac{\lambda}{\beta}\left\{t+\frac{1}{\Gamma(\beta)}\right\}^{\beta}\right]\right. \\
& \left. \pm \sqrt{m} \operatorname{sn}\left[\frac{-\iota x}{\sqrt{(m-6 \sqrt{m}+1) \rho}}-\frac{\lambda}{\beta}\left\{t+\frac{1}{\Gamma(\beta)}\right\}^{\beta}\right]\right) .
\end{aligned}
$$

Set 6:

$$
\left\{\alpha_{-1}=\mp i \sqrt{\frac{2 \rho \lambda}{\theta}} \sqrt[4]{\frac{-1}{(m-6 \sqrt{m}+1) \rho}}, \alpha_{0}=0, \alpha_{1}= \pm i \sqrt{\frac{2 m \rho \lambda}{\theta}} \sqrt[4]{\frac{-1}{(m-6 \sqrt{m}+1) \rho}}, \omega=\frac{-\iota}{\sqrt{(m-6 \sqrt{m}+1) \rho}}\right\} .
$$

By using the Eqs. (13) and (24) into Eq. (9), we get

$$
\begin{aligned}
q(x, t) & =i \sqrt{\frac{2 \rho \lambda}{\theta}} \sqrt[4]{\frac{-1}{(m-6 \sqrt{m}+1) \rho}}\left(\mp \operatorname{sn}^{-1}\left[\frac{-\iota x}{\sqrt{(m-6 \sqrt{m}+1) \rho}}-\frac{\lambda}{\beta}\left\{t+\frac{1}{\Gamma(\beta)}\right\}^{\beta}\right]\right. \\
& \left. \pm \sqrt{m} \operatorname{sn}\left[\frac{-\iota x}{\sqrt{(m-6 \sqrt{m}+1) \rho}}-\frac{\lambda}{\beta}\left(t+\frac{1}{\Gamma(\beta)}\right)^{\beta}\right]\right) .
\end{aligned}
$$

Set 7:

$$
\left\{\alpha_{-1}=-\sqrt{\frac{2 \lambda \rho}{\theta}} \sqrt[4]{\frac{-1}{(m+6 \sqrt{m}+1) \rho}}, \alpha_{0}=0, \alpha_{1}=-\sqrt{\frac{2 \lambda m \rho}{\theta}} \sqrt[4]{\frac{-1}{(m+6 \sqrt{m}+1) \rho}}, \omega=\frac{-\iota}{\sqrt{(m+6 \sqrt{m}+1) \rho}}\right\} .
$$


By using Eqs. (13) and (26) into Eq. (9), we get

$$
\begin{aligned}
q(x, t) & =-\sqrt{\frac{2 \lambda \rho}{\theta}} \sqrt[4]{\frac{-1}{(m+6 \sqrt{m}+1) \rho}}\left(\operatorname{sn}^{-1}\left[\frac{-\iota x}{\sqrt{(m+6 \sqrt{m}+1) \rho}}-\frac{\lambda}{\beta}\left\{t+\frac{1}{\Gamma(\beta)}\right\}^{\beta}\right]\right. \\
& \left.+\sqrt{m} \operatorname{sn}\left[\frac{-\iota x}{\sqrt{(m+6 \sqrt{m}+1) \rho}}-\frac{\lambda}{\beta}\left\{t+\frac{1}{\Gamma(\beta)}\right\}^{\beta}\right]\right) .
\end{aligned}
$$

\section{Set 8:}

$$
\left\{\alpha_{-1}=\sqrt{\frac{2 \lambda \rho}{\theta}} \sqrt[4]{-\frac{1}{(m+6 \sqrt{m}+1) \rho}}, \alpha_{0}=0, \alpha_{1}=\sqrt{\frac{2 \lambda m \rho}{\theta}} \sqrt[4]{\frac{-1}{(m+6 \sqrt{m}+1) \rho}}, \omega=\frac{-\iota}{\sqrt{(m+6 \sqrt{m}+1) \rho}}\right\}
$$

By using Eqs. (13) and (28) into Eq. (9), we obtain

$$
\begin{aligned}
q(x, t) & =\sqrt{\frac{2 \lambda \rho}{\theta}} \sqrt[4]{\frac{-1}{(m+6 \sqrt{m}+1) \rho}}\left(\operatorname{sn}^{-1}\left[\frac{-\iota x}{\sqrt{(m+6 \sqrt{m}+1) \rho}}-\frac{\lambda}{\beta}\left\{t+\frac{1}{\Gamma(\beta)}\right\}^{\beta}\right]\right. \\
& \left.+\sqrt{m} \operatorname{sn}\left[\frac{-\iota x}{\sqrt{(m+6 \sqrt{m}+1) \rho}}-\frac{\lambda}{\beta}\left\{t+\frac{1}{\Gamma(\beta)}\right\}^{\beta}\right]\right) .
\end{aligned}
$$

Set 9:

$$
\left\{\alpha_{-1}=-i \sqrt{\frac{2 \lambda \rho}{\theta}} \sqrt[4]{\frac{-1}{(m+6 \sqrt{m}+1) \rho}}, \alpha_{0}=0, \alpha_{1}=-\sqrt{\frac{2 \lambda m \rho}{\theta}} \sqrt[4]{\frac{-1}{(m+6 \sqrt{m}+1) \rho}}, \omega=\frac{\iota}{\sqrt{(m+6 \sqrt{m}+1) \rho}}\right\}
$$

By using Eqs. (13) and (30) into Eq. (9), yields

$$
\begin{aligned}
q(x, t) & =-i \sqrt{\frac{2 \lambda \rho}{\theta}} \sqrt[4]{\frac{-1}{(m+6 \sqrt{m}+1) \rho}}\left(\operatorname{sn}^{-1}\left[\frac{\iota x}{\sqrt{(m+6 \sqrt{m}+1) \rho}}-\frac{\lambda}{\beta}\left\{t+\frac{1}{\Gamma(\beta)}\right\}^{\beta}\right]\right. \\
& \left.+\sqrt{m} \operatorname{sn}\left[\frac{\iota x}{\sqrt{(m+6 \sqrt{m}+1) \rho}}-\frac{\lambda}{\beta}\left\{t+\frac{1}{\Gamma(\beta)}\right\}^{\beta}\right]\right) .
\end{aligned}
$$

\section{Set 10:}

$$
\left\{\alpha_{-1}=i \sqrt{\frac{2 \lambda \rho}{\theta}} \sqrt[4]{\frac{-1}{(m+6 \sqrt{m}+1) \rho}}, \alpha_{0}=0, \alpha_{1}=i \sqrt{\frac{2 \lambda m \rho}{\theta}} \sqrt[4]{\frac{-1}{(m+6 \sqrt{m}+1) \rho}}, \omega=\frac{\iota}{\sqrt{(m+6 \sqrt{m}+1) \rho}}\right\}
$$

By using Eqs. (13) and (32) into Eq. (9), we get

$$
\begin{aligned}
q(x, t) & =i \sqrt{\frac{2 \lambda \rho}{\theta}} \sqrt[4]{\frac{-1}{(m+6 \sqrt{m}+1) \rho}}\left(\operatorname{sn}^{-1}\left[\frac{\iota x}{\sqrt{(m+6 \sqrt{m}+1) \rho}}-\frac{\lambda}{\beta}\left\{t+\frac{1}{\Gamma(\beta)}\right\}^{\beta}\right]\right. \\
& \left.+\sqrt{m} \operatorname{sn}\left[\frac{\iota x}{\sqrt{(m+6 \sqrt{m}+1) \rho}}-\frac{\lambda}{\beta}\left\{t+\frac{1}{\Gamma(\beta)}\right\}^{\beta}\right]\right) .
\end{aligned}
$$




\subsection{Dark, singular and combined soliton solutions}

When $m \rightarrow 1$ then from the solution for $q(x, t)$ for each set, the dark and singular soliton solutions given as:

$$
\begin{aligned}
& q(x, t)=\mp \frac{\sqrt{2} \sqrt{\lambda} \sqrt{\rho}}{\sqrt{\theta} \sqrt[4]{-2 \rho}} \operatorname{coth}\left(\frac{-x}{\sqrt{-2 \rho}}-\frac{\lambda}{\beta}\left[t+\frac{1}{\Gamma(\beta)}\right]^{\beta}\right) . \\
& q(x, t)=\mp \frac{\sqrt{2} \sqrt{\lambda} \sqrt{\rho}}{\sqrt{\theta} \sqrt[4]{-2 \rho}} \tanh \left(\frac{-x}{\sqrt{-2 \rho}}-\frac{\lambda}{\beta}\left[t+\frac{1}{\Gamma(\beta)}\right]^{\beta}\right), \\
& q(x, t)=\mp \frac{i \sqrt{2} \sqrt{\lambda} \sqrt{\rho}}{\sqrt{\theta} \sqrt[4]{-2 \rho}} \operatorname{coth}\left(\frac{x}{\sqrt{-2 \rho}}-\frac{\lambda}{\beta}\left[t+\frac{1}{\Gamma(\beta)}\right]^{\beta}\right) \text {, } \\
& q(x, t)=\mp \frac{i \sqrt{2} \sqrt{\lambda} \sqrt{\rho}}{\sqrt{\theta} \sqrt[4]{-2 \rho}} \tanh \left(\frac{x}{\sqrt{-2 \rho}}-\frac{\lambda}{\beta}\left[t+\frac{1}{\Gamma(\beta)}\right]^{\beta}\right), \\
& q(x, t)=\sqrt{\frac{2 \rho \lambda}{\theta}} \sqrt[4]{\frac{1}{4 \rho}}\left(\mp \operatorname{coth}\left[\frac{-\iota x}{\sqrt{-4 \rho}}-\frac{\lambda}{\beta}\left\{t+\frac{1}{\Gamma(\beta)}\right\}^{\beta}\right] \pm \tanh \left[\frac{-\iota x}{\sqrt{-4 \rho}}-\frac{\lambda}{\beta}\left\{t+\frac{1}{\Gamma(\beta)}\right\}^{\beta}\right]\right), \\
& q(x, t)=i \sqrt{\frac{2 \rho \lambda}{\theta}} \sqrt[4]{\frac{1}{4 \rho}}\left(\mp \operatorname{coth}\left[\frac{-\iota x}{\sqrt{-4 \rho}}-\frac{\lambda}{\beta}\left\{t+\frac{1}{\Gamma(\beta)}\right\}^{\beta}\right] \pm \tanh \left[\frac{-\iota x}{\sqrt{-4 \rho}}-\frac{\lambda}{\beta}\left\{t+\frac{1}{\Gamma(\beta)}\right\}^{\beta}\right]\right), \\
& q(x, t)=-\sqrt{\frac{2 \lambda \rho}{\theta}} \sqrt[4]{\frac{-1}{8 \rho}}\left(\operatorname{coth}\left[\frac{-\iota x}{\sqrt{8 \rho}}-\frac{\lambda}{\beta}\left\{t+\frac{1}{\Gamma(\beta)}\right\}^{\beta}\right]+\tanh \left[\frac{-\iota x}{\sqrt{8 \rho}}-\frac{\lambda}{\beta}\left\{t+\frac{1}{\Gamma(\beta)}\right\}^{\beta}\right]\right), \\
& q(x, t)=\sqrt{\frac{2 \lambda \rho}{\theta}} \sqrt[4]{\frac{-1}{8 \rho}}\left(\operatorname{coth}\left[\frac{-\iota x}{\sqrt{8 \rho}}-\frac{\lambda}{\beta}\left\{t+\frac{1}{\Gamma(\beta)}\right\}^{\beta}\right]+\tanh \left[\frac{-\iota x}{\sqrt{8 \rho}}-\frac{\lambda}{\beta}\left\{t+\frac{1}{\Gamma(\beta)}\right\}^{\beta}\right]\right), \\
& q(x, t)=-i \sqrt{\frac{2 \lambda \rho}{\theta}} \sqrt[4]{\frac{-1}{8 \rho}}\left(\operatorname{coth}\left[\frac{\iota x}{\sqrt{8 \rho}}-\frac{\lambda}{\beta}\left\{t+\frac{1}{\Gamma(\beta)}\right\}^{\beta}\right]+\tanh \left[\frac{\iota x}{\sqrt{8 \rho}}-\frac{\lambda}{\beta}\left\{t+\frac{1}{\Gamma(\beta)}\right\}^{\beta}\right]\right) \\
& q(x, t)=i \sqrt{\frac{2 \lambda \rho}{\theta}} \sqrt[4]{\frac{-1}{8 \rho}}\left(\operatorname{coth}\left[\frac{\iota x}{\sqrt{8 \rho}}-\frac{\lambda}{\beta}\left\{t+\frac{1}{\Gamma(\beta)}\right\}^{\beta}\right]+\tanh \left[\frac{\iota x}{\sqrt{8 \rho}}-\frac{\lambda}{\beta}\left\{t+\frac{1}{\Gamma(\beta)}\right\}^{\beta}\right]\right) .
\end{aligned}
$$

Case 2: If $Y=Y(\xi)=c n(\xi, m)$ Eq. (12) becomes

$$
Q(\eta)=\alpha_{-1} c n^{-1}(\eta, m)+\alpha_{0}+\alpha_{1} c n(\eta, m) .
$$

By plugging Eq. (44) into the Eq. (11), we get the below solution sets:

\section{Set 1:}

$$
\left\{\alpha_{-1}=\mp i \sqrt{2} \sqrt{\frac{\lambda(m-1) \rho}{\theta \sqrt{(2 m-1) \rho}}}, \alpha_{0}=0, \alpha_{1}=0, \omega=\frac{-1}{\sqrt{(2 m-1) \rho}}\right\} .
$$

By using Eqs. (44) and (45) into Eq. (9), we get

$$
q(x, t)=\mp i \sqrt{2} \sqrt{\frac{\lambda(m-1) \rho}{\theta \sqrt{(2 m-1) \rho}}} \mathrm{cn}^{-1}\left(\frac{-x}{\sqrt{(2 m-1) \rho}}-\frac{\lambda}{\beta}\left[t+\frac{1}{\Gamma(\beta)}\right]^{\beta}\right) .
$$

Set 2:

$$
\left\{\alpha_{-1}=0, \alpha_{0}=0, \alpha_{1}=\mp \frac{i \sqrt{2 \lambda m \rho}}{\sqrt{\theta} \sqrt[4]{(2 m-1) \rho}}, \omega=\frac{-1}{\sqrt{(2 m-1) \rho}}\right\} .
$$

By using Eqs. (44) and (47) into Eq. (9), we get

$$
q(x, t)=\mp \frac{i \sqrt{2 \lambda m \rho}}{\sqrt{\theta} \sqrt[4]{(2 m-1) \rho}} \operatorname{cn}\left(\frac{-x}{\sqrt{(2 m-1) \rho}}-\frac{\lambda}{\beta}\left[t+\frac{1}{\Gamma(\beta)}\right]^{\beta}\right) .
$$


Set 3:

$$
\left\{\alpha_{-1}=\mp \sqrt{2} \sqrt{\frac{\lambda(m-1) \rho}{\theta \sqrt{(2 m-1) \rho}}}, \alpha_{0}=0, \alpha_{1}=0, \omega=\frac{1}{\sqrt{(2 m-1) \rho}}\right\} .
$$

By using Eqs. (44) and (49) into Eq. (9), we get

Set 4:

$$
q(x, t)=\mp \sqrt{2} \sqrt{\frac{\lambda(m-1) \rho}{\theta \sqrt{(2 m-1) \rho}}} \mathrm{cn}^{-1}\left(\frac{x}{\sqrt{(2 m-1) \rho}}-\frac{\lambda}{\beta}\left[t+\frac{1}{\Gamma(\beta)}\right]^{\beta}\right) .
$$

$$
\left\{\alpha_{-1}=0, \alpha_{0}=0, \alpha_{1}=\mp \frac{\sqrt{2 \lambda m \rho}}{\sqrt{\theta} \sqrt[4]{(2 m-1) \rho}}, \omega=\frac{1}{\sqrt{(2 m-1) \rho}}\right\} .
$$

By using Eqs. (44) and (52) into Eq. (9), we get

$$
q(x, t)=\mp \frac{\sqrt{2 \lambda m \rho}}{\sqrt{\theta} \sqrt[4]{(2 m-1) \rho}} \mathrm{cn}\left(\frac{x}{\sqrt{(2 m-1) \rho}}-\frac{\lambda}{\beta}\left[t+\frac{1}{\Gamma(\beta)}\right]^{\beta}\right) .
$$

Set 5:

$$
\begin{aligned}
& \left\{\alpha_{-1}=\mp i \sqrt{2} \sqrt{\frac{\lambda(m-1) \sqrt{\frac{-6 \sqrt{(m-1) m \rho^{2}}-2 m \rho+\rho}{32 m^{2}-32 m-1}}}{\theta}}, \quad \alpha_{0}=0,\right. \\
& \left.\alpha_{1}= \pm i \sqrt{2 m} \sqrt{\frac{\lambda \sqrt{\frac{-6 \sqrt{(m-1) m \rho^{2}}-2 m \rho+\rho}{32 m^{2}-32 m-1}}}{\theta}}, \quad \omega=-\sqrt{\frac{-2 m-6 \sqrt{(m-1) m}+1}{\left(32 m^{2}-32 m-1\right) \rho}}\right\} .
\end{aligned}
$$

By using Eqs. (44) and (54) into Eq. (9), we have

$$
\begin{aligned}
q(x, t) & =i \sqrt{2} \sqrt{\frac{\lambda \sqrt{\frac{-6 \sqrt{(m-1) m \rho^{2}}-2 m \rho+\rho}{32 m^{2}-32 m-1}}}{\theta}}\left(\mp \sqrt{m-1} \mathrm{cn}^{-1}\left[-x \sqrt{\frac{-2 m-6 \sqrt{(m-1) m}+1}{\left(32 m^{2}-32 m-1\right) \rho}-\frac{\lambda}{\beta}\left\{t+\frac{1}{\Gamma(\beta)}\right.}\right\}^{\beta}\right] \\
& \left. \pm \sqrt{m} \mathrm{cn}\left[-x \sqrt{\frac{-2 m-6 \sqrt{(m-1) m}+1}{\left(32 m^{2}-32 m-1\right) \rho}}-\frac{\lambda}{\beta}\left\{t+\frac{1}{\Gamma(\beta)}\right\}^{\beta}\right]\right)
\end{aligned}
$$

Set 6:

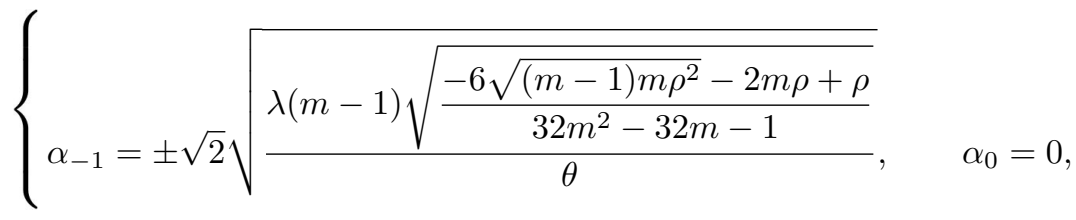

$$
\begin{aligned}
& \begin{array}{l}
\left.\alpha_{1}=\mp \sqrt{2 m} \sqrt{\frac{\lambda \sqrt{\frac{-6 \sqrt{(m-1) m \rho^{2}}-2 m \rho+\rho}{32 m^{2}-32 m-1}}}{\theta}}, \quad \omega=\sqrt{\frac{-2 m-6 \sqrt{(m-1) m}+1}{\left(32 m^{2}-32 m-1\right) \rho}}\right\} . \\
\text { (44) and (55) into Eq. (9), we get }
\end{array}
\end{aligned}
$$

By using Eqs. (44) and (55) into Eq. (9), we get

$$
\begin{aligned}
q(x, t) & =\sqrt{2} \sqrt{\frac{\lambda \sqrt{\frac{-6 \sqrt{(m-1) m \rho^{2}}-2 m \rho+\rho}{32 m^{2}-32 m-1}}}{\theta}}\left( \pm \sqrt{m-1} \mathrm{cn}^{-1}\left[x \sqrt{\frac{-2 m-6 \sqrt{(m-1) m}+1}{\left(32 m^{2}-32 m-1\right) \rho}-\frac{\lambda}{\beta}\left\{t+\frac{1}{\Gamma(\beta)}\right.}\right\}^{\beta}\right] \\
& \left.\mp \sqrt{m} \mathrm{cn}\left[x \sqrt{\frac{-2 m-6 \sqrt{(m-1) m}+1}{\left(32 m^{2}-32 m-1\right) \rho}}-\frac{\lambda}{\beta}\left\{t+\frac{1}{\Gamma(\beta)}\right\}^{\beta}\right]\right)
\end{aligned}
$$


Set 7:

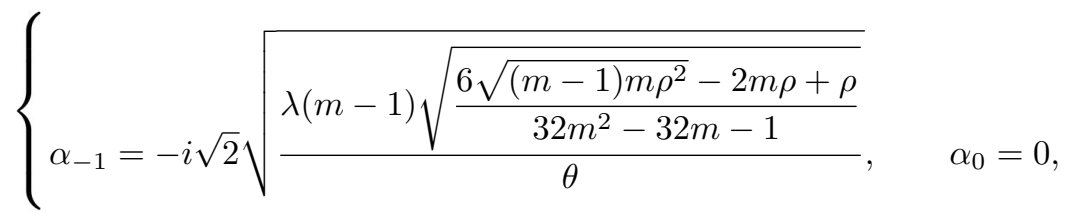

$$
\begin{aligned}
& \left.\alpha_{1}=-i \sqrt{2 m} \sqrt{\frac{\lambda \sqrt{\frac{6 \sqrt{(m-1) m \rho^{2}}-2 m \rho+\rho}{32 m^{2}-32 m-1}}}{\theta}}, \quad \omega=-\sqrt{\frac{-2 m+6 \sqrt{(m-1) m}+1}{\left(32 m^{2}-32 m-1\right) \rho}}\right\} .
\end{aligned}
$$

By using Eqs. (44) and (57) into Eq. (9), we get

$$
\begin{aligned}
q(x, t) & =-i \sqrt{\frac{2 \lambda \sqrt{\frac{6 \sqrt{(m-1) m \rho^{2}}-2 m \rho+\rho}{32 m^{2}-32 m-1}}}{\theta}}\left(\sqrt{m-1} \mathrm{cn}^{-1}\left[-x \sqrt{\frac{-2 m+6 \sqrt{(m-1) m}+1}{\left(32 m^{2}-32 m-1\right) \rho}-\frac{\lambda}{\beta}\left\{t+\frac{1}{\Gamma(\beta)}\right.}\right\}^{\beta}\right] \\
& \left.+\sqrt{m} \mathrm{cn}\left[-x \sqrt{\frac{-2 m+6 \sqrt{(m-1) m}+1}{\left(32 m^{2}-32 m-1\right) \rho}}-\frac{\lambda}{\beta}\left\{t+\frac{1}{\Gamma(\beta)}\right\}^{\beta}\right]\right)
\end{aligned}
$$

Set 8:

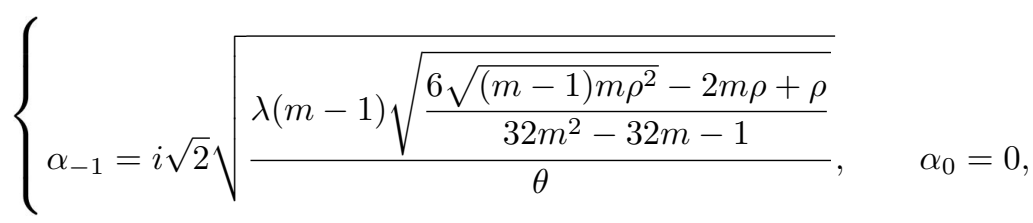

$$
\begin{aligned}
& \left.\alpha_{1}=i \sqrt{2 m} \sqrt{\frac{\lambda \sqrt{\frac{6 \sqrt{(m-1) m \rho^{2}}-2 m \rho+\rho}{32 m^{2}-32 m-1}}}{\theta}}, \quad \omega=-\sqrt{\frac{-2 m+6 \sqrt{(m-1) m}+1}{\left(32 m^{2}-32 m-1\right) \rho}}\right\} .
\end{aligned}
$$

By using Eqs. (44) and (59) into Eq. (9), we get

$$
\begin{aligned}
q(x, t) & =i \sqrt{2} \sqrt{\frac{\lambda \sqrt{\frac{6 \sqrt{(m-1) m \rho^{2}}-2 m \rho+\rho}{32 m^{2}-32 m-1}}}{\theta}}\left(\sqrt { m - 1 } \mathrm { cn } ^ { - 1 } \left[-x \sqrt{\left.\frac{-2 m+6 \sqrt{(m-1) m}+1}{\left(32 m^{2}-32 m-1\right) \rho}-\frac{\lambda}{\beta}\left\{t+\frac{1}{\Gamma(\beta)}\right\}^{\beta}\right]}\right.\right. \\
& \left.+\sqrt{m} \mathrm{cn}\left[-x \sqrt{\frac{-2 m+6 \sqrt{(m-1) m}+1}{\left(32 m^{2}-32 m-1\right) \rho}}-\frac{\lambda}{\beta}\left\{t+\frac{1}{\Gamma(\beta)}\right\}^{\beta}\right]\right) .
\end{aligned}
$$

Set 9:

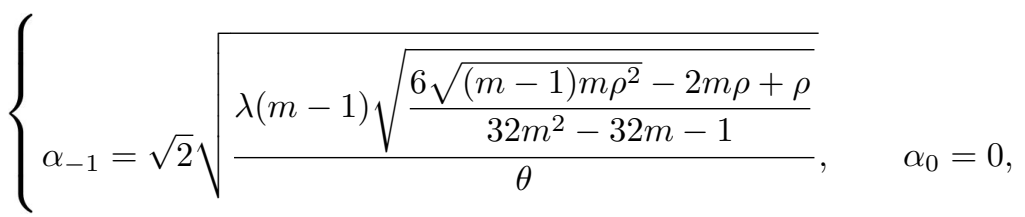

$$
\begin{aligned}
& \left.\alpha_{1}=\sqrt{2 m} \sqrt{\frac{\lambda \sqrt{\frac{6 \sqrt{(m-1) m \rho^{2}}-2 m \rho+\rho}{32 m^{2}-32 m-1}}}{\theta}}, \quad \omega=\sqrt{\frac{-2 m+6 \sqrt{(m-1) m}+1}{\left(32 m^{2}-32 m-1\right) \rho}}\right\} .
\end{aligned}
$$


By using Eqs. (44) and (61) into Eq. (9), we get

$$
\begin{aligned}
q(x, t) & =\sqrt{2} \sqrt{\frac{\lambda \sqrt{\frac{6 \sqrt{(m-1) m \rho^{2}}-2 m \rho+\rho}{32 m^{2}-32 m-1}}}{\theta}}\left(\sqrt { m - 1 } \mathrm { cn } ^ { - 1 } \left[x \sqrt{\left.\frac{-2 m+6 \sqrt{(m-1) m}+1}{\left(32 m^{2}-32 m-1\right) \rho}-\frac{\lambda}{\beta}\left\{t+\frac{1}{\Gamma(\beta)}\right\}^{\beta}\right]}\right.\right. \\
& \left.+\sqrt{m} \mathrm{cn}\left[x \sqrt{\frac{-2 m+6 \sqrt{(m-1) m}+1}{\left(32 m^{2}-32 m-1\right) \rho}}-\frac{\lambda}{\beta}\left\{t+\frac{1}{\Gamma(\beta)}\right\}^{\beta}\right]\right)
\end{aligned}
$$

Set 10:

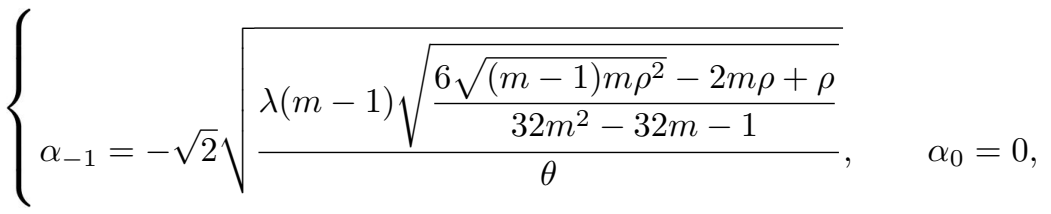

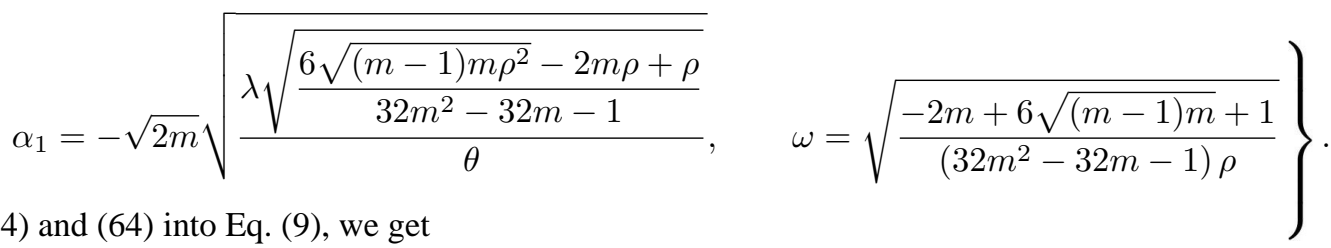

By using Eqs. (44) and (64) into Eq. (9), we get

$$
\begin{aligned}
q(x, t) & =-\sqrt{2} \sqrt{\frac{\lambda \sqrt{\frac{6 \sqrt{(m-1) m \rho^{2}}-2 m \rho+\rho}{32 m^{2}-32 m-1}}}{\theta}}\left(\sqrt { m - 1 } \mathrm { cn } ^ { - 1 } \left[x \sqrt{\left.\frac{-2 m+6 \sqrt{(m-1) m}+1}{\left(32 m^{2}-32 m-1\right) \rho}-\frac{\lambda}{\beta}\left\{t+\frac{1}{\Gamma(\beta)}\right\}^{\beta}\right]}\right.\right. \\
& \left.+\sqrt{m} \mathrm{cn}\left[x \sqrt{\frac{-2 m+6 \sqrt{(m-1) m}+1}{\left(32 m^{2}-32 m-1\right) \rho}}-\frac{\lambda}{\beta}\left\{t+\frac{1}{\Gamma(\beta)}\right\}^{\beta}\right]\right) .
\end{aligned}
$$

\subsection{Bright soliton solutions:}

When $m \rightarrow 1$ then the bright soliton solutions above reduce to

$$
\begin{aligned}
& q(x, t)=\mp \frac{i \sqrt{2 \lambda \rho}}{\sqrt{\theta} \sqrt[4]{\rho}} \operatorname{sech}\left(\frac{-x}{\sqrt{\rho}}-\frac{\lambda}{\beta}\left[t+\frac{1}{\Gamma(\beta)}\right]^{\beta}\right), \\
& q(x, t)=\mp \frac{\sqrt{2 \lambda \rho}}{\sqrt{\theta} \sqrt[4]{\rho}} \operatorname{sech}\left(\frac{x}{\sqrt{\rho}}-\frac{\lambda}{\beta}\left[t+\frac{1}{\Gamma(\beta)}\right]^{\beta}\right), \\
& q(x, t)= \pm i \sqrt{\frac{2 \lambda \sqrt{\rho}}{\theta}} \operatorname{sech}\left(\frac{-x}{\sqrt{\rho}}-\frac{\lambda}{\beta}\left[t+\frac{1}{\Gamma(\beta)}\right]^{\beta}\right) .
\end{aligned}
$$

Case 3: If $Y=Y(\xi)=d n(\xi, m)$ Eq. (12) becomes:

$$
Q(\eta)=\alpha_{-1} d n^{-1}(\eta, m)+\alpha_{0}+\alpha_{1} d n(\eta, m) .
$$

By putting Eq. (68) into the Eq. (11), we get the solution sets given by

Set 1:

$$
\left\{\alpha_{-1}=\mp \sqrt{2} \sqrt{\frac{\lambda(m-1) \rho}{\theta \sqrt{-(m-2) \rho}}}, \quad \alpha_{0}=0, \quad \alpha_{1}=0, \quad \omega=\frac{-1}{\sqrt{-(m-2) \rho}}\right\} .
$$

By using Eqs. (68) and (69) into Eq. (9), we get

$$
q(x, t)=\mp \sqrt{2} \sqrt{\frac{\lambda(m-1) \rho}{\theta \sqrt{-(m-2) \rho}}} \mathrm{dn}^{-1}\left(\frac{-x}{\sqrt{-(m-2) \rho}}-\frac{\lambda}{\beta}\left[t+\frac{1}{\Gamma(\beta)}\right]^{\beta}\right) .
$$


Set 2:

By using Eqs. (68) and (71) into Eq. (9), we get

$$
\left\{\alpha_{-1}=0, \quad \alpha_{0}=0, \quad \alpha_{1}=\mp \frac{i \sqrt{2 \lambda \rho}}{\sqrt{\theta} \sqrt[4]{-(m-2) \rho}}, \quad \omega=\frac{-1}{\sqrt{-(m-2) \rho}}\right\} .
$$

$$
q(x, t)=\mp \frac{i \sqrt{2 \lambda \rho}}{\sqrt{\theta} \sqrt[4]{-(m-2) \rho}} \operatorname{dn}\left(\frac{-x}{\sqrt{-(m-2) \rho}}-\frac{\lambda}{\beta}\left[t+\frac{1}{\Gamma(\beta)}\right]^{\beta}\right) .
$$

Set 3:

$$
\left\{\alpha_{-1}=\mp \iota \sqrt{2} \sqrt{\frac{\lambda(m-1) \rho}{\theta \sqrt{-(m-2) \rho}}}, \quad \alpha_{0}=0, \quad \alpha_{1}=0, \quad \omega=\frac{1}{\sqrt{-(m-2) \rho}}\right\} .
$$

By using Eqs. (68) and (73) into Eq. (9), we get

$$
q(x, t)=\mp \iota \sqrt{2} \sqrt{\frac{\lambda(m-1) \rho}{\theta \sqrt{-(m-2) \rho}}} \mathrm{dn}^{-1}\left(\frac{x}{\sqrt{-(m-2) \rho}}-\frac{\lambda}{\beta}\left[t+\frac{1}{\Gamma(\beta)}\right]^{\beta}\right) .
$$

Set 4:

$$
\left\{\alpha_{-1}=0, \quad \alpha_{0}=0, \quad \alpha_{1}=\mp \frac{\sqrt{2 \lambda \rho}}{\sqrt{\theta} \sqrt[4]{-(m-2) \rho}}, \quad \omega=\frac{1}{\sqrt{-(m-2) \rho}}\right\} .
$$

By using Eqs. (68) and (75) into Eq. (9), we get

$$
q(x, t)=\mp \frac{\sqrt{2 \lambda \rho}}{\sqrt{\theta} \sqrt[4]{-(m-2) \rho}} \operatorname{dn}\left(\frac{x}{\sqrt{-(m-2) \rho}}-\frac{\lambda}{\beta}\left[t+\frac{1}{\Gamma(\beta)}\right]^{\beta}\right) .
$$

Set 5:

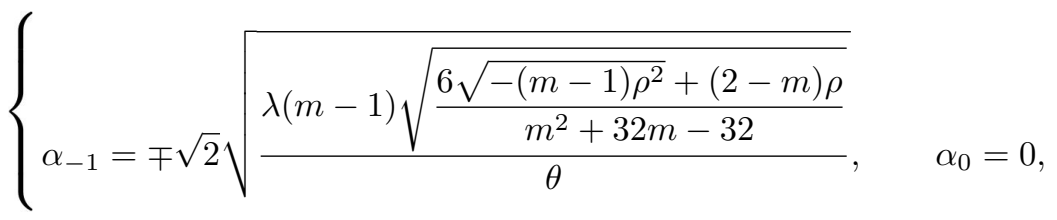

$$
\begin{aligned}
& \left.\alpha_{1}=\mp \iota \sqrt{2} \sqrt{\frac{\lambda \sqrt{\frac{6 \sqrt{-(m-1) \rho^{2}}+(2-m) \rho}{m^{2}+32 m-32}}}{\theta}}, \quad \omega=-\sqrt{\frac{-m+6 \sqrt{-(m-1)}+2}{\left(m^{2}+32 m-32\right) \rho}}\right\} .
\end{aligned}
$$

By using Eqs. (68) and (77) into Eq. (9), we get

$$
\begin{aligned}
q(x, t) & =\mp \sqrt{2} \sqrt{\frac{\lambda \sqrt{\frac{6 \sqrt{-(m-1) \rho^{2}}+(2-m) \rho}{m^{2}+32 m-32}}}{\theta}}\left(\sqrt { m - 1 } \mathrm { dn } ^ { - 1 } \left[-x \sqrt{\left.\frac{-m+6 \sqrt{-(m-1)}+2}{\left.m^{2}+32 m-32\right) \rho}-\frac{\lambda}{\beta}\left\{t+\frac{1}{\Gamma(\beta)}\right\}^{\beta}\right]}\right.\right. \\
& \left.+\iota \operatorname{dn}\left[-x \sqrt{\frac{-m+6 \sqrt{-(m-1)}+2}{\left(m^{2}+32 m-32\right) \rho}}-\frac{\lambda}{\beta}\left\{t+\frac{1}{\Gamma(\beta)}\right\}^{\beta}\right]\right) .
\end{aligned}
$$

Set 6:

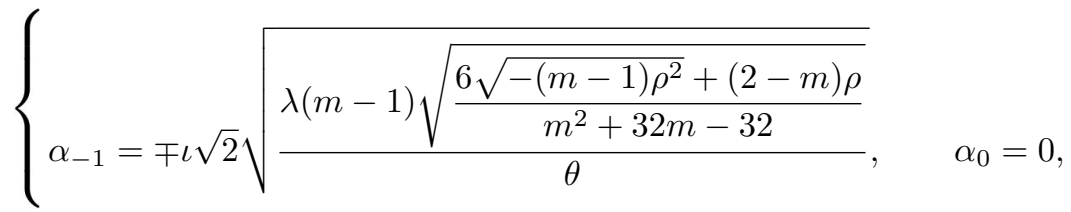

$$
\begin{aligned}
& \left.\alpha_{1}= \pm \sqrt{2} \sqrt{\frac{\lambda \sqrt{\frac{6 \sqrt{-(m-1) \rho^{2}}+(2-m) \rho}{m^{2}+32 m-32}}}{\theta}}, \quad \omega=\sqrt{\frac{(2-m)+6 \sqrt{-(m-1)}}{\left(m^{2}+32 m-32\right) \rho}}\right\} .
\end{aligned}
$$


By using Eqs. (68) and (79) into Eq. (9), we get

$$
\begin{aligned}
q(x, t) & =\sqrt{2} \sqrt{\frac{\lambda \sqrt{\frac{6 \sqrt{-(m-1) \rho^{2}}+(2-m) \rho}{m^{2}+32 m-32}}}{\theta}}\left(\mp \iota \sqrt { m - 1 } \mathrm { dn } ^ { - 1 } \left[x \sqrt{\left.\frac{(2-m)+6 \sqrt{-(m-1)}}{\left(m^{2}+32 m-32\right) \rho}-\frac{\lambda}{\beta}\left\{t+\frac{1}{\Gamma(\beta)}\right\}^{\beta}\right]}\right.\right. \\
& \pm \operatorname{dn}\left[x \sqrt{\frac{(2-m)+6 \sqrt{-(m-1)}}{\left(m^{2}+32 m-32\right) \rho}}-\frac{\lambda}{\beta}\left\{t+\frac{1}{\Gamma(\beta)}\right\}^{\beta}\right] .
\end{aligned}
$$

Set 7:

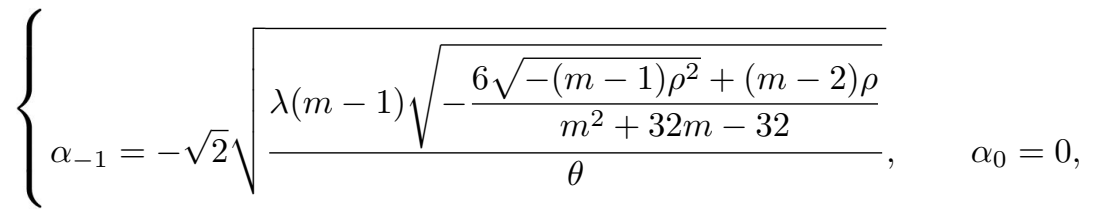

$$
\begin{aligned}
& \begin{array}{l}
\left.\alpha_{1}=\sqrt{2} \iota \sqrt{\frac{\lambda \sqrt{-\frac{6 \sqrt{-(m-1) \rho^{2}}+(m-2) \rho}{m^{2}+32 m-32}}}{\theta}}, \quad \omega=-\sqrt{-\frac{(m-2)+6 \sqrt{-(m-1)}}{\left(m^{2}+32 m-32\right) \rho}}\right\} . \\
\text { 68) and (81) into Eq. (9), we get }
\end{array}
\end{aligned}
$$

By using Eqs. (68) and (81) into Eq. (9), we get

$$
\begin{aligned}
q(x, t) & =\sqrt{2} \sqrt{\frac{\lambda \sqrt{-\frac{6 \sqrt{-(m-1) \rho^{2}}+(m-2) \rho}{m^{2}+32 m-32}}}{\theta}}\left(-\sqrt{m-1} \mathrm{dn}^{-1}\left[-x \sqrt{\left.-\frac{(m-2)+6 \sqrt{-(m-1)}}{\left(m^{2}+32 m-32\right) \rho}-\frac{\lambda}{\beta}\left\{t+\frac{1}{\Gamma(\beta)}\right\}^{\beta}\right]}\right.\right. \\
& \left.+\iota \mathrm{dn}\left[-x \sqrt{-\frac{(m-2)+6 \sqrt{-(m-1)}}{\left(m^{2}+32 m-32\right) \rho}}-\frac{\lambda}{\beta}\left\{t+\frac{1}{\Gamma(\beta)}\right\}^{\beta}\right]\right)
\end{aligned}
$$

Set 8:

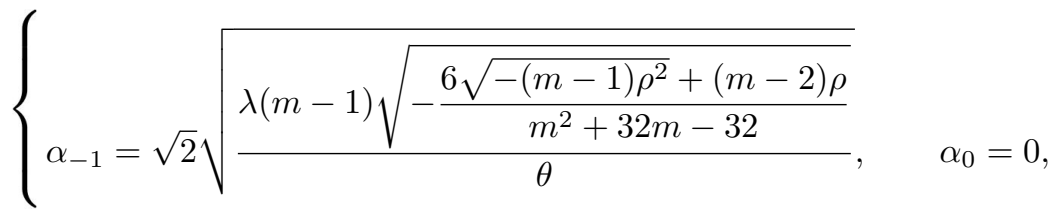

$$
\begin{aligned}
& \begin{array}{l}
\left.\alpha_{1}=-\iota \sqrt{2} \sqrt{\frac{\lambda \sqrt{-\frac{6 \sqrt{-(m-1) \rho^{2}}+(m-2) \rho}{m^{2}+32 m-32}}}{\theta}}, \quad \omega=-\sqrt{-\frac{(m-2)+6 \sqrt{-(m-1)}}{\left(m^{2}+32 m-32\right) \rho}}\right\} . \\
\text { (68) and (83) into Eq. (9), we get }
\end{array}
\end{aligned}
$$

By using Eqs. (68) and (83) into Eq. (9), we get

$$
\begin{aligned}
q(x, t) & =\sqrt{2} \sqrt{\frac{\lambda \sqrt{-\frac{6 \sqrt{-(m-1) \rho^{2}}+(m-2) \rho}{m^{2}+32 m-32}}}{\theta}}\left(\sqrt { m - 1 } \operatorname { d n } ^ { - 1 } \left(\left[-x \sqrt{\left.-\frac{(m-2)+6 \sqrt{-(m-1)}}{\left(m^{2}+32 m-32\right) \rho}-\frac{\lambda}{\beta}\left\{t+\frac{1}{\Gamma(\beta)}\right\}^{\beta}\right]}\right.\right.\right. \\
& \left.-\iota \operatorname{dn}\left[-x \sqrt{-\frac{(m-2)+6 \sqrt{-(m-1)}}{\left(m^{2}+32 m-32\right) \rho}}-\frac{\lambda}{\beta}\left\{t+\frac{1}{\Gamma(\beta)}\right\}^{\beta}\right]\right)
\end{aligned}
$$

Set 9: 


$$
\begin{aligned}
& \left\{\alpha_{-1}=-\sqrt{2} \iota \sqrt{\frac{\lambda(m-1) \sqrt{-\frac{6 \sqrt{-(m-1) \rho^{2}}+(m-2) \rho}{m^{2}+32 m-32}}}{\theta}}, \quad \alpha_{0}=0\right. \\
& \left.\alpha_{1}=-\sqrt{2} \sqrt{\frac{\lambda \sqrt{-\frac{6 \sqrt{-(m-1) \rho^{2}}+(m-2) \rho}{m^{2}+32 m-32}}}{\theta}}, \quad \omega=\sqrt{-\frac{(m-2)+6 \sqrt{-(m-1)}}{\left(m^{2}+32 m-32\right) \rho}}\right\} . \\
& q(x, t)=-\sqrt{2} \sqrt{\frac{\lambda \sqrt{-\frac{6 \sqrt{-(m-1) \rho^{2}}+(m-2) \rho}{m^{2}+32 m-32}}}{\theta}}\left(\iota \sqrt{m-1} \mathrm{dn}^{-1}\left[x \sqrt{-\frac{(m-2)+6 \sqrt{-(m-1)}}{\left(m^{2}+32 m-32\right) \rho}-\frac{\lambda}{\beta}\left\{t+\frac{1}{\Gamma(\beta)}\right.}\right\}^{\beta}\right] \\
& \left.+\operatorname{dn}\left[x \sqrt{-\frac{(m-2)+6 \sqrt{-(m-1)}}{\left(m^{2}+32 m-32\right) \rho}}-\frac{\lambda}{\beta}\left\{t+\frac{1}{\Gamma(\beta)}\right\}^{\beta}\right]\right) \text {. }
\end{aligned}
$$

Set 10:

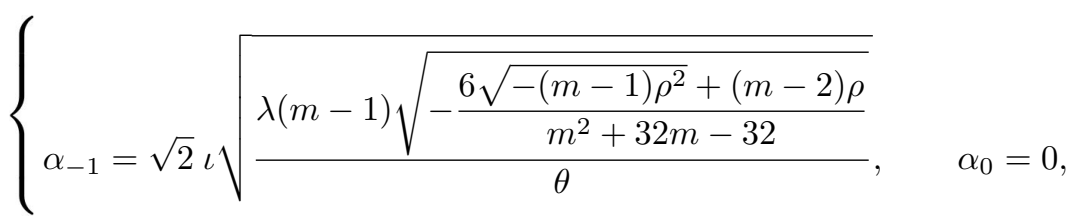

$$
\begin{aligned}
& \begin{array}{l}
\alpha_{1}=\sqrt{2} \sqrt{\frac{\lambda \sqrt{-\frac{6 \sqrt{-(m-1) \rho^{2}}+(m-2) \rho}{m^{2}+32 m-32}}}{\theta}}, \quad \omega=\sqrt{-\frac{(m-2)+6 \sqrt{-(m-1)}}{\left(m^{2}+32 m-32\right) \rho}} \\
\text { (68) and (87) into Eq. (9), we get }
\end{array}
\end{aligned}
$$

By using Eqs. (68) and (87) into Eq. (9), we get

$$
\begin{aligned}
q(x, t) & =\sqrt{2} \sqrt{\frac{\lambda \sqrt{-\frac{6 \sqrt{-(m-1) \rho^{2}}+(m-2) \rho}{m^{2}+32 m-32}}}{\theta}}\left(\iota \sqrt { m - 1 } \mathrm { dn } ^ { - 1 } \left[x \sqrt{\left.-\frac{(m-2)+6 \sqrt{-(m-1)}}{\left(m^{2}+32 m-32\right) \rho}-\frac{\lambda}{\beta}\left\{t+\frac{1}{\Gamma(\beta)}\right\}^{\beta}\right]}\right.\right. \\
& \left.+\operatorname{dn}\left[x \sqrt{-\frac{(m-2)+6 \sqrt{-(m-1)}}{\left(m^{2}+32 m-32\right) \rho}}-\frac{\lambda}{\beta}\left\{t+\frac{1}{\Gamma(\beta)}\right\}^{\beta}\right]\right)
\end{aligned}
$$

When $m \rightarrow 1$ then the solutions above allow for the bright soliton solution given by we get the bright soliton solutions given as:

$$
q(x, t)= \pm \sqrt{\frac{2 \lambda}{\theta} \sqrt{\rho}} \operatorname{sech}\left(\frac{x}{\rho}-\frac{\lambda}{\beta}\left[t+\frac{1}{\Gamma(\beta)}\right]^{\beta}\right) .
$$

\subsection{Bright and singular soliton solutions with the Kudryashov Method}

By applying the homogenous balance technique between the terms $Q^{3}$ and $Q^{\prime \prime}$ into Eq. (11), we have $m=1$. For $m=1$, Eq. (5) reduces to

$$
Q(\eta)=\alpha_{0}+\alpha_{1} \phi(\eta)
$$

Here $\alpha_{0}$ and $\alpha_{1}$ are unknown constants. By inserting Eqs. (6) and (90) into Eq. (11) and collecting the all coefficients of same order of $\phi(\eta)$, we get the algebraic expressions involving $\alpha_{0}, \alpha_{1}$ and other parameters. Now using Mathemat ica,

Set 1:

$$
\left\{\alpha_{0}=0, \quad \alpha_{1}=\mp \frac{i \sqrt{2} \sqrt{d} \sqrt{\lambda} \sqrt[4]{\rho}}{\sqrt{\theta}}, \quad \omega=-\frac{1}{\sqrt{\rho}}\right\} .
$$


By using Eqs. (90) and (91) into Eq. (9), we get

$$
q(x, t)=\mp \frac{i \sqrt{2} \sqrt{d} \sqrt{\lambda} \sqrt[4]{\rho}}{\sqrt{\theta}\left([a-b] \sinh \left[\omega x-\frac{\lambda}{\beta}\left\{t+\frac{1}{\Gamma(\beta)}\right\}^{\beta}\right]+[a+b] \cosh \left[\omega x-\frac{\lambda}{\beta}\left\{t+\frac{1}{\Gamma(\beta)}\right\}^{\beta}\right]\right)} .
$$

Set 2:

$$
\left\{\alpha_{0}=0, \alpha_{1}=\mp \frac{\sqrt{2} \sqrt{d} \sqrt{\lambda} \sqrt[4]{\rho}}{\sqrt{\theta}}, \omega=\frac{1}{\sqrt{\rho}}\right\} .
$$

By using Eqs. (90) and (93) into Eq. (9), we get

$$
q(x, t)=\mp \frac{\sqrt{2} \sqrt{d} \sqrt{\lambda} \sqrt[4]{\rho}}{\sqrt{\theta}\left([a-b] \sinh \left[\omega x-\frac{\lambda}{\beta}\left\{t+\frac{1}{\Gamma(\beta)}\right\}^{\beta}\right]+[a+b] \cosh \left[\omega x-\frac{\lambda}{\beta}\left\{t+\frac{1}{\Gamma(\beta)}\right\}^{\beta}\right]\right)} .
$$

\section{Conclusion}

We have successfully attempted to produce a variety of soliton-type solutions including the dark, bright, singular and other types of solitons for the fractional MEW equation using a beta-time derivative. The required results have been obtained by applying the extended Jacobi's elliptic expansion function method and the Kudryashov method. The secured results have been verified through symbolic soft computations. The equation in this investigation has been considered for the first time in the Beta derivative sense via the above-mentioned approaches and the solutions can potentially be helpful for further development in this field.

1. A. Biswas and R. T. Alqahtani, Chirp-free bright optical solitons for perturbed Gerdjikov-Ivanov equation by semi-inverse variational principle, Optik 147 (2017) 72, https://doi. org/10.1016/j.ijleo.2017.08.019.

2. A. Bekir, Applications of the extended tanh method for coupled nonlinear evolution equations, Commun. Nonlinear Sci. Numer. Simul. 13 (2008) 1748, https: //doi.org/10.1016/j. cnsns.2007.05.001

3. H. Rezazadeh et al., Hyperbolic rational solutions to a variety of conformable fractional Boussinesq-like equations, Nonlinear Eng. 8 (2019) 224, https://doi.org/10.1515/ nleng-2018-0033

4. J.-H. He, Variational principle and periodic solution of the Kundu-Mukherjee-Naskar equation, Res. Phys. 17 (2020) 103031, https://doi.org/10.1016/j.rinp.2020. 103031 .

5. Y. Yıldırım, and M. Mirzazadeh, Optical pulses with KunduMukherjee-Naskar model in fiber communication systems, Chin. J. Phys. 64 (2020) 183, https://doi.org/10. 1016/j.cjph.2019.10.025

6. B. A. Malomed, D. Mihalache, F. Wise, and L. Torner, Spatiotemporal optical solitons, J. Opt. B 7 (2005) R53, https: //doi.org/10.1088/1464-4266/7/5/R02

7. A. Zafar, M. Raheel, and A. Bekir, Exploring the dark and singular soliton solutions of Biswas-Arshed model with full nonlinear form, Optik 204 (2020) 164133, https : //doi.org/ $10.1016 / j . i j l e o .2019 .164133$
8. W. Malfliet, Solitary wave solutions of nonlinear wave equations, Am. J. Phys. 60 (1992) 650, https: // doi.org/10 . $1119 / 1.17120$

9. K. R. Raslan, K. K. Ali, and M. A. Shallal, The modified extended tanh method with the Riccati equation for solving the space-time fractional EW and MEW equations, Chaos Solitons Fractals 103 (2017) 404, https://doi.org/10.1016/ j.chaos.2017.06.029

10. D. Shi and Y. Zhang, Diversity of exact solutions to the conformable space-time fractional MEW equation, Appl. Math. Lett. 99 (2020) 105994, https://doi.org/10.1016/j. aml.2019.07.025.

11. A. Biswas, M. Ekici, A. Sonmezoglu, and M. R. Belic, Highly dispersive optical solitons with cubic-quintic-septic law by extended Jacobi's elliptic function expansion, $O p$ tik 183 (2019) 571, https://doi.org/10.1016/j. ijleo.2019.02.127

12. A. Biswas, M. Ekici, A. Sonmezoglu, and M. R. Belic, Highly dispersive optical solitons with Kerr law nonlinearity by extended Jacobi's elliptic function expansion, $O p$ tik 183 (2019) 395, https://doi.org/10.1016/j. ijleo.2019.02.050

13. M. A. Abdou and A. Elhanbaly, Construction of periodic and solitary wave solutions by the extended Jacobi elliptic function expansion method, Commun. Nonlinear Sci. Numer. Simul. 12 (2007) 1229, https://doi.org/10.1016/j.cnsns. 2006.01 .013 
14. H. Zhang, Extended Jacobi elliptic function expansion method and its applications, Commun. Nonlinear Sci. Numer. Simul. 12 (2007) 627, https://doi.org/10.1016/j.cnsns. 2005.08 .003

15. W. Zhang, Extended Jacobi Elliptic Function Expansion Method to the ZK-MEW Equation, Int. J. Differ. Equ. 2011 (2011) 451420, https://doi.org/10.1155/2011/ 451420

16. R. I. Nuruddeen and A. M. Nass, Exact solitary wave solution for the fractional and classical GEW-Burgers equations: an application of Kudryashov method, J. Taibah Univ. Sci. 12 (2018) 309, https://doi.org/10.1080/16583655. 2018.1469283

17. K. Hosseini, M. Mirzazadeh, M. Ilie, and S. Radmehr, Dynamics of optical solitons in the perturbed Gerdjikov-Ivanov equation, Optik 206 (2020) 164350, https://doi.org/ $10.1016 / j . i j l e o .2020 .164350$

18. K. Hosseini, and Z. Ayati, Exact solutions of space-time fractional EW and modified EW equations using Kudryashov method, Nonlinear Sci. Lett. A 7 (2016) 58.

19. N. A. Kudryashov, Method for finding highly dispersive optical solitons of nonlinear differential equations, Optik 206 (2020) 163550, https://doi.org/10.1016/j. ijleo.2019.163550

20. K. Hosseini, M. Mirzazadeh, M. Ilie, and J. F. Gómez-Aguilar, Biswas-Arshed equation with the beta time derivative: Optical solitons and other solutions, Optik 217 (2020) 164801, https://doi.org/10.1016/j.ijleo.2020. 164801

21. K. Hosseini, M. Mirzazadeh, and J. F. Gómez-Aguilar, Soliton solutions of the Sasa-Satsuma equation in the monomode optical fibers including the beta-derivatives, Optik 224 (2020) 165425, https://doi.org/10.1016/j. ijleo.2020.165425

22. H. Yépes-Martínez, J. F. Gómez-Aguilar, and D. Baleanu, Beta-derivative and subequation method applied to the optical solitons in medium with parabolic law nonlinearity and higher order dispersion, Optik 155 (2018) 357, https:// doi.org/10.1016/j.ijleo.2017.10.104
23. H. Yépez-Martínez and J. F. Gómez-Aguilar, Fractional subequation method for Hirota-Satsuma-coupled $\mathrm{KdV}$ equation and coupled mKdV equation using the Atangana's conformable derivative, Waves Random Complex Media 29 (2019) 678, https://doi.org/10.1080/17455030.2018. 1464233

24. H. Yépez-Martínez and J. F. Gómez-Aguilar, Optical solitons solution of resonance nonlinear Schrödinger type equation with Atangana's-conformable derivative using subequation method, Waves Random Complex Media 31 (2021) 573, https:// doi.org/10.1080/17455030.2019.1603413

25. A. Atangana, D. Baleanu and A. Alsaedi, Analysis of timefractional Hunter-Saxton equation: a model of neumatic liquid crystal, Open Phys. 14 (2016) 145, https://doi.org/ $10.1515 /$ phys-2016-0010

26. A. Atangana and R. T. Alqahtani, Modelling the Spread of River Blindness Disease via the Caputo Fractional Derivative and the Beta-derivative, Entropy 18 (2016) 40, https: //doi.org/10.3390/e18020040

27. H. Yépez-Martínez, J.F. Gómez-Aguilar and D. Baleanu, Betaderivative and sub-equation method applied to the optical solitons in medium with parabolic law nonlinearity and higher order dispersion, Optik. 155 (2018) 357.

28. A. Yusuf, M. Inc, A. I. Aliyu, and D. Baleanu, Optical Solitons Possessing Beta Derivative of the Chen-Lee-Liu Equation in Optical Fibers, Front. Phys. 7 (2019) 34, https: //doi.org/10.3389/fphy.2019.00034

29. M. F. Uddin, M. Golam Hafez, Z. Hammouch, and D. Baleanu, Periodic and rogue waves for Heisenberg models of ferromagnetic spin chains with fractional beta derivative evolution and obliqueness, Waves Random Complex Media (in press), https://doi.org/10.1080/17455030. 2020.1722331.

30. B. Ghanbari and J. F. Gómez-Aguilar, The generalized exponential rational function method for Radhakrishnan-KunduLakshmanan equation with $\beta$-conformable time derivative, Rev. Mex. Fis. 65 (2019) 503, https://doi.org/10.31349/ RevMexFis.65.503 